\title{
Factores predictores de la satisfacción de estudiantes de cursos virtuales
}

\section{Prediction factors of student satisfaction in online courses}

\author{
Jimmy Zambrano Ramírez \\ Instituto Superior Tecnológico Rumiñahui (Ecuador)
}

\section{Resumen}

La facilidad de acceso y uso cotidiano de las recientes tecnologías de la información y la comunicación ha permitido un desarrollo impresionante de ofertas universitarias completamente virtuales. Estos desarrollos también han planteado importantes cuestiones sobre cuáles son los determinantes que afectan el aprendizaje, el desempeño y la retención de los estudiantes de estas ofertas académicas. Uno de estos determinantes es el grado en el cual los cursos o programas virtuales satisfacen las expectativas de los estudiantes. En este estudio se investigó los factores predictores de la satisfacción estudiantil identificados por Sun y sus colaboradores (2008) con estudiantes hispanohablantes. Se tradujo el cuestionario al español y fue respondido por 102 participantes. El análisis de consistencia interna dio como resultado alta confiabilidad. El análisis de correlación mostró que todos los factores estudiados, con excepción de la ansiedad por el uso de computadoras, están significativamente correlacionados con la satisfacción estudiantil. El análisis de regresión con pasos sucesivos encontró que los factores flexibilidad del curso, actitud docente hacia el e-learning, autoeficacia del estudiante en el uso de Internet y percepción de la interacción predicen el $47.2 \%$ de la satisfacción estudiantil. Basado en estos resultados se ofrecen orientaciones para los administradores de cursos virtuales de educación superior.

Palabras clave: satisfacción del alumnado; aprendizaje asistido por ordenador; enseñanza a distancia; administración educativa.

\begin{abstract}
The easy access and daily use of recent information and communication technologies has led to an impressive development of university offers completely in online modality. These developments have also raised important questions about the determining factors that affect learning, performance and retention of the learners of these academic programs. One of these determinants is the degree to which virtual courses or programs satisfy the learners' expectations. Specifically, this study investigated the predictor factors of learner satisfaction identified by Sun and colleagues (2008) among Hispanic learners. The questionnaire was translated into Spanish and filled out by 102 participants. The internal consistency analysis resulted in high reliability. Correlation analysis showed that all the factors studied, except computer anxiety, are significantly correlated with learner satisfaction. The stepwise regression analysis found that course flexibility, instructor attitude towards e-learning, student Internet
\end{abstract}


self-efficacy, and perception of the interaction factors determine almost $47.2 \%$ of student satisfaction. Based on these results, guidance is offered for managers of higher education virtual programs.

Keywords: student satisfaction; computer-based learning; distance teaching; educational administration.

El creciente interés de los centros universitarios en la educación virtual así como la introducción de las tecnologías en los procesos de instrucción y de aprendizaje plantean importantes desafíos y preguntas para quienes diseñan políticas educativas institucionales, para los administradores educativos, los instructores, e incluso para los mismos estudiantes (Zambrano, 2012). Ante esto, la investigación científica está produciendo muchísimas orientaciones sobre los diferentes aspectos y factores relacionados con la implementación efectiva de programas virtuales, tal como se puede observar en la reciente literatura científica (Moore, 2013; Rudestam y Schoenholtz-Read, 2010). Sin embargo, aún existen muchas preguntas por clarificar. Una de estas está relacionada con la satisfacción de los estudiantes con respecto a su experiencia de aprender a través de cursos virtuales.

\section{LA SATISFACCIÓN ESTUDIANTIL}

La satisfacción estudiantil ha sido concebida como el grado de congruencia entre las expectativas previas de los estudiantes y los resultados obtenidos, con respecto a la experiencia de aprender a través de cursos virtuales (Allen, Omori, Burrell, Mabry y Timmerman, 2013; Moore y Shelton, 2014; Simpson, 2003). Se la considera como un estándar clave para la evaluación de la calidad de la educación virtual (Rubio Gómez, 2003). Además, se ha sugerido que la satisfacción está asociada al desempeño y la retención de los estudiantes en los cursos virtuales, pero la evidencia empírica sobre esto todavía no es concluyente (Drouin, 2008). De hecho, se ha encontrado que la satisfacción estudiantil está mediada por factores que no están asociados directamente con el curso o la institución educativa (Peralta Castro, Escobar Jurado, Mora Rodríguez, Martínez González y Rocío Velandia, 2014).

La búsqueda de evidencias de factores asociados o que predicen la satisfacción estudiantil ha dado lugar a numerosos estudios empíricos, e incluso a algunos metaanálisis. Estas investigaciones han identificado factores tales como:

- $\quad$ el instructor, la tecnología y la interactividad (Bolliger y Martindale, 2004);

- la interacción de los estudiantes con el contenido, con los profesores y con los otros estudiantes (Swan, 2001); 
- el sentido de comunidad (Drouin, 2008), la perseverancia en desafíos de estudios independientes, la facilidad de uso del computador y de interacción con el docente (Bray, Aoki, y Dlugosh, 2008);

- la presencia de enseñanza, cognitiva y social (Akyol y Garrison, 2010);

- la ansiedad del estudiante, la actitud del profesor, la flexibilidad, la calidad del curso, la percepción de utilidad, la facilidad de uso del sistema y la diversidad de la evaluación, (Sun, Tsai, Finger, Chen, y Yeh, 2008);

- la organización del curso, del proceso de enseñanza-aprendizaje y de las instalaciones e infraestructura (Sanjuán Gómez, Gómez Martínez, Rabell Piera, Arcia Arcia, y Morales Velázquez, 2011).

Meta-análisis como el de Allen, Bourhis, Burrell y Mabry (2002) y el de Williams (2006) dan luces sobre qué factores predicen la satisfacción estudiantil en la educación virtual, e incluso comparan sus resultados con los de la educación presencial.

Estos estudios pueden orientar la evaluación de la satisfacción estudiantil de los programas virtuales para hispanohablantes. No obstante, a la hora de emprender un proceso de evaluación nos encontramos con algunos problemas: la mayoría de las investigaciones publicadas han sido hechas entre poblaciones anglohablantes; hay muchos factores o variables relacionadas con la satisfacción estudiantil, de las cuales algunos términos se refieren a una misma variable (e.g., interactividad e interacción estudiantes-estudiantes) y diferentes variables son nombradas con un mismo término (e.g., docente, instructor, o facilitador); además, existen instrumentos para diferentes factores que se miden con diversas escalas. Debido a estos problemas, se precisa de un modelo que integre los factores que afectan la satisfacción en un solo instrumento y cuyos componentes hayan mostrado tener evidencia empírica según la literatura científica.

En la revisión de la literatura, se pudo identificar al modelo de Sun et al. (2008) como uno de los que podría superar los problemas arriba mencionados. Sun y sus colaboradores han tenido en cuenta las múltiples dimensiones con las que debe abordarse la satisfacción estudiantil. Por esto, su modelo está compuesto por seis dimensiones y trece variables o factores independientes y la satisfacción estudiantil como variable dependiente. La dimensión estudiante está compuesta por los factores: actitud hacia las computadoras, ansiedad y autoeficacia; la dimensión instructor por: respuesta oportuna y actitud hacia el e-learning; la dimensión curso por: flexibilidad y calidad; la dimensión tecnología por: calidad tecnológica y calidad de la Internet; la dimensión diseño por: percepción de utilidad y de facilidad de uso del sistema; y la dimensión ambiente por: diversidad de la evaluación del aprendizaje y percepción de interacción con otros.

El modelo de Sun y sus colaboradores tiene algunas ventajas. Se puede observar que las dimensiones y factores que lo componen se basan en evidencia empírica. 
Además, el instrumento ha sido validado, tiene alta confiabilidad y se ha aplicado a estudiantes universitarios. Su limitación es que aún no ha sido probado con poblaciones de hispanohablantes. Por esto, no se sabe cuán confiable es el instrumento en este contexto, qué variables tienen mayor o menor capacidad predictiva, o qué variables tienen más relevancia según el dominio específico de conocimiento o carrera universitaria. Para superar esta limitación, se requiere traducir y adaptar el instrumento, llevar a cabo algunas investigaciones que arrojen luces sobre la pertinencia de los factores originalmente planteados, así como mejorar el modelo introduciendo otros factores y condiciones asociadas a la satisfacción estudiantil.

Por consiguiente, en este estudio se dará el paso inicial de probar el instrumento con estudiantes hispanohablantes y confirmar las hipótesis planteadas por Sun et al. (2008). Para esto, se consideran todas las dimensiones, factores y reactivos tal y como fueron establecidos en el estudio original. Véase la tabla 1. Al aplicar el instrumento será posible identificar las variables que de forma individual están correlacionadas significativamente y cuáles de ellas, al combinarse, predicen mejor la satisfacción. Esto es, la presente investigación permitirá observar si los factores que predicen la satisfacción de estudiantes taiwaneses también predicen la satisfacción de sus homólogos ecuatorianos; y observar sus diferencias. Consecuentemente, utilizaremos las mismas hipótesis:

H1. La actitud de los estudiantes hacia las computadoras está positivamente relacionada con su percepción de satisfacción.

H2. La ansiedad de los estudiantes por el uso de computadoras está negativamente relacionada con su percepción de satisfacción.

H3. La autoeficacia de los estudiantes por el uso de Internet está positivamente relacionada con su percepción de satisfacción.

H4. La respuesta oportuna del docente está positivamente relacionada con la percepción de satisfacción estudiantil.

H5. Las actitudes del docente hacia el e-learning están positivamente relacionadas con la percepción de satisfacción estudiantil.

H6. La flexibilidad del curso está positivamente relacionada con la percepción de satisfacción estudiantil.

H7. La calidad del curso está positivamente relacionada con la percepción de satisfacción estudiantil.

H8. La calidad de la tecnología está positivamente relacionada con la percepción de satisfacción estudiantil.

H9. La calidad de la Internet está positivamente relacionada con la percepción de satisfacción estudiantil.

H10. La percepción de utilidad del sistema está positivamente relacionada con la percepción de satisfacción estudiantil. 
H11. La percepción de facilidad de uso del sistema está positivamente relacionada con la percepción de satisfacción estudiantil.

H12. La diversidad de la evaluación del aprendizaje está positivamente relacionada con la percepción de satisfacción estudiantil.

H13. La percepción de la interacción con otros está positivamente relacionada con su percepción de satisfacción.

Tabla 1. Dimensiones, factores y reactivos del cuestionario de la satisfacción estudiantil

\begin{tabular}{|l|l|}
\hline \multicolumn{1}{|c|}{ Factores } & \multicolumn{1}{c|}{ Reactivos } \\
\hline \multicolumn{1}{|c|}{$\begin{array}{l}\text { Estoy satisfecho con mi decisión de tomar este curso por Internet. } \\
\text { Si tengo la oportunidad de tomar otro curso vía Internet, con gusto lo } \\
\text { haría. } \\
\text { Fue sabia mi elección de tomar este curso por Internet. } \\
\text { Me quedé satisfecho con este curso. } \\
\text { Siento que este curso satisfizo mis necesidades. } \\
\text { Voy a tomar tantos cursos por Internet como pueda. } \\
\text { Quedé decepcionado por cómo se llevó a cabo este curso. (I) } \\
\text { Si tuviera que hacerlo de nuevo, no tomaría este curso por Internet. (I) } \\
\text { Se me ha hecho más difícil tomar este curso a través de Internet que otros } \\
\text { cursos. (I) }\end{array}$} \\
\hline $\begin{array}{l}\text { Factor 1. } \\
\text { Actitud de los } \\
\text { estudiantes } \\
\text { hacia las } \\
\text { computadoras }\end{array}$ & $\begin{array}{l}\text { Creo que trabajar con computadoras es difícil. (I) } \\
\text { Creo que trabajar con computadoras es muy complicado. (I) } \\
\text { Creo que trabajar con computadoras requiere habilidades técnicas. (I) } \\
\text { Creo que trabajar con computadoras me hace sentir gran tensión } \\
\text { psicológica. (I) } \\
\text { programación (tales como el Bue trabajar con computadoras es recomendable para personas con } \\
\text { mucha paciencia. (I) } \\
\text { Creo que trabajar con computadoras hace a una persona más productiva } \\
\text { en su trabajo. } \\
\text { Creo que trabajar con computadoras es solo para personas jóvenes. (I) }\end{array}$ \\
\hline $\begin{array}{l}\text { Factor 2. } \\
\text { Ansiedad del } \\
\text { estudiante } \\
\text { por el uso de } \\
\text { computadoras }\end{array}$ & $\begin{array}{l}\text { Trabajar con una computadora me hace sentir muy nervioso. } \\
\text { Me da angustia cuando pienso que tengo que utilizar una computadora. } \\
\text { Las computadoras me hacen sentir incómodo. }\end{array}$ \\
\hline
\end{tabular}




\begin{tabular}{|c|c|}
\hline $\begin{array}{l}\text { Factor } 3 . \\
\text { Autoeficacia } \\
\text { del estudiante } \\
\text { en el uso de } \\
\text { Internet }\end{array}$ & $\begin{array}{l}\text { Siento confianza para iniciar un curso (materia o asignatura) a través de la } \\
\text { conexión a Internet. } \\
\text { Siento confianza en conectarme a las páginas de Internet que desee. } \\
\text { Siento confianza en finalizar este curso a través de Internet. } \\
\text { Siento confianza en descargar los materiales de aprendizaje (textos, audio, } \\
\text { vídeos, etc.) desde Internet. } \\
\text { Siento confianza en dar clic en las páginas de Internet que desee. } \\
\text { Siento confianza en ir a las páginas anteriores dando clic en la función } \\
\text { "Atrás". } \\
\text { Siento confianza en ir a la siguiente página dando clic en la función } \\
\text { "Adelante". } \\
\text { Siento confianza al desplazarme en la pantalla de la computadora. } \\
\text { Siento confianza en Internet para hacer búsquedas en Google, Yahoo u } \\
\text { otros. } \\
\text { Siento confianza en encontrar en Internet la información que necesito para } \\
\text { un tema específico. } \\
\text { Siento confianza en seleccionar las palabras correctas para buscar en } \\
\text { Internet. } \\
\text { Siento confianza en imprimir materiales de Internet. } \\
\text { Siento confianza en finalizar todo el programa de estudios por Internet. }\end{array}$ \\
\hline \multicolumn{2}{|r|}{ Dimensión 2: Docente } \\
\hline $\begin{array}{l}\text { Factor 4: } \\
\text { Respuesta } \\
\text { oportuna del } \\
\text { docente }\end{array}$ & $\begin{array}{l}\text { Recibí comentarios sobre mis actividades o tareas del curso de forma } \\
\text { oportuna. }\end{array}$ \\
\hline $\begin{array}{l}\text { Factor } 5: \\
\text { Actitud del } \\
\text { docente hacia } \\
\text { el e-learning }\end{array}$ & $\begin{array}{l}\text { Comparando tu experiencia de aprendizaje entre clases presenciales } \\
\text { y virtuales, ¿piensas que el profesor de este curso utiliza la web } \\
\text { adecuadamente para enseñar? }\end{array}$ \\
\hline \multicolumn{2}{|r|}{ Dimensión 3: Curso } \\
\hline $\begin{array}{l}\text { Factor 6: } \\
\text { Flexibilidad del } \\
\text { curso }\end{array}$ & $\begin{array}{l}\text { Tomar este curso por Internet me permitió organizar mis tareas de clases } \\
\text { más efectivamente. } \\
\text { Las ventajas de tomar clases por Internet superan cualquier desventaja. } \\
\text { Tomar este curso por Internet me permitió tener más tiempo para otras } \\
\text { actividades. } \\
\text { No hubo serios inconvenientes en tomar el curso por Internet. } \\
\text { Tomar este curso por Internet me permitió organizar mi horario de trabajo } \\
\text { de forma más efectiva. } \\
\text { Tomar este curso por Internet me permitió ahorrar el tiempo de transporte } \\
\text { que implica ir a una clase presencial. } \\
\text { Tomé este curso por Internet porque de otra manera no hubiera sido } \\
\text { posible. } \\
\text { Tomar cursos por Internet me permitirá terminar el programa de estudio } \\
\text { más rápidamente. }\end{array}$ \\
\hline
\end{tabular}




\begin{tabular}{|c|c|}
\hline $\begin{array}{l}\text { Factor 7: } \\
\text { Calidad del } \\
\text { curso }\end{array}$ & $\begin{array}{l}\text { El desarrollo del curso a través de Internet ha mejorado su calidad en } \\
\text { comparación con otros cursos. } \\
\text { La calidad de este curso se compara favorablemente con la de mis otros } \\
\text { cursos. } \\
\text { La calidad del curso no fue afectada por utilizar la Internet. }\end{array}$ \\
\hline \multicolumn{2}{|r|}{ Dimensión 4: Tecnológica } \\
\hline $\begin{array}{l}\text { Factor 8: } \\
\text { Calidad } \\
\text { tecnológica }\end{array}$ & $\begin{array}{l}\text { Siento que las tecnologías de la información (computadoras, celulares, } \\
\text { conexión a Internet, etc.) utilizadas en los cursos por Internet son muy } \\
\text { fáciles de usar. } \\
\text { Siento que las tecnologías de la información empleadas en los cursos por } \\
\text { Internet tienen muchas funciones útiles. } \\
\text { Siento que las tecnologías de la información usadas en los cursos por } \\
\text { Internet son flexibles. } \\
\text { Siento que las tecnologías de la información utilizadas en los cursos por } \\
\text { Internet son fáciles de adquirir. }\end{array}$ \\
\hline $\begin{array}{l}\text { Factor 9: } \\
\text { Calidad de } \\
\text { Internet }\end{array}$ & $\begin{array}{l}\text { Estoy satisfecho con la velocidad de mi conexión a Internet. } \\
\text { Siento que la calidad de comunicación por Internet no es buena. (I) } \\
\text { Siento que el pago de la conexión a Internet es muy caro. (I) } \\
\text { Siento que es fácil estar conectado a Internet. }\end{array}$ \\
\hline \multicolumn{2}{|r|}{ Dimensión 5: Diseño } \\
\hline $\begin{array}{l}\text { Factor 10: } \\
\text { Percepción de } \\
\text { utilidad del } \\
\text { sistema virtual }\end{array}$ & $\begin{array}{l}\text { El uso de las plataformas web (tales como Itslearning o Moodle) } \\
\text { aumentará mi efectividad en el programa de estudios. } \\
\text { El uso de las plataformas web mejorará mi desempeño en el programa de } \\
\text { estudios. } \\
\text { Las plataformas web son útiles para el programa de estudios. } \\
\text { El uso de las plataformas web aumentará mi productividad. }\end{array}$ \\
\hline $\begin{array}{l}\text { Factor 11: } \\
\text { Percepción de } \\
\text { facilidad de } \\
\text { uso del sistema } \\
\text { virtual }\end{array}$ & $\begin{array}{l}\text { Me es fácil desempeñarme bien en el manejo de las plataformas web. } \\
\text { Me es fácil aprender a utilizar las plataformas web. } \\
\text { Me es fácil conseguir una plataforma web para hacer lo que yo quiera. } \\
\text { Me es fácil usar una plataforma web. }\end{array}$ \\
\hline \multicolumn{2}{|r|}{ Dimensión 6: Ambiental } \\
\hline $\begin{array}{l}\text { Factor 12: } \\
\text { Diversidad en } \\
\text { la evaluación }\end{array}$ & $\begin{array}{l}\text { Este curso ofrece muchas formas de evaluación de mi aprendizaje } \\
\text { (cuestionarios, trabajos escritos, presentaciones orales, etc.). }\end{array}$ \\
\hline
\end{tabular}


La interacción entre estudiantes fue más difícil que en otros cursos. (I) Es difícil participar en las discusiones en este curso, en comparación con otros cursos. (I)

Aprendí más de mis compañeros de este curso que en otros cursos. El instructor animó frecuentemente a que los estudiantes interactúen.

Factor 13: Percepción de la interacción con otros Mientras el curso progresó, la interacción con otros estudiantes y el profesor, a través de la plataforma web, fue más natural.

Sentí que la calidad de las discusiones, a través de la plataforma, fue alta durante todo el curso.

Fue fácil seguir el hilo de las discusiones a través de la plataforma web. Las dinámicas de clase (discusiones grupales, lluvia de ideas, juego de roles, etc.) no fueron tan diferentes a las de otros cursos.

No se utilizó la plataforma para interactuar con los demás estudiantes de la clase. (I)

Nota: Cuestionario traducido de "What drives a successful e-learning? An empirical investigation of the critical factors influencing learner satisfaction," por P.-C. Sun, R. J. Tsai, G. Finger, Y-Y. Chen y D. Yeh, 2008, Computers \& Education, 5O, pp. 1197-1199. Se usa una escala tipo Likert de 7 puntos ordenada desde 'totalmente en desacuerdo' (marcada con 1) a 'totalmente de acuerdo' (marcada con 7) para medir todos los reactivos del cuestionario. Los marcados con (I) denota que se miden con la escala inversa.

Fuente: Traducción realizada por autor

\section{METODOLOGÍA}

\section{Participantes}

Los participantes fueron estudiantes $(N=102)$, hombres $(N=65)$ y mujeres $(N$ = 37) matriculados en el programa de Teología modalidad virtual de la Fundación FLEREC. Los participantes equivalen al 81.6\% del total de 125 estudiantes matriculados en el programa. Se aseguró a los participantes que su contribución en la investigación era confidencial.

El programa de teología dura cuatro años, los cuales se cursan en dieciséis trimestres. Los estudiantes tienen la exigencia de tomar cuatro cursos en cada trimestre. El $75 \%$ del programa se compone de cursos con contenido conceptual predominante, tales como Teología sistemática, Antiguo y Nuevo Testamento, Historia de la iglesia cristiana europea y latinoamericana; el $25 \%$ restante, de cursos con contenido procedimental predominante, tales como Consejería Pastoral y Didáctica de la educación religiosa.

Todos los cursos del programa de teología están soportados en una plataforma web. Los materiales de los cursos requieren que los estudiantes aprendan principalmente de lecturas de artículos y libros electrónicos, guías de estudio escritas y explicaciones didácticas redactadas por los docentes. La comunicación entre los participantes 
ocurre a través de foros de discusión, de la mensajería de la plataforma y de mensajes de correo electrónico. Las tareas de aprendizaje fomentan la comprensión, el análisis y la reflexión crítica, así como la aplicación de los conocimientos para resolver los problemas de las comunidades eclesiásticas de los estudiantes.

\section{Procedimiento}

El cuestionario de factores críticos de Sun et al. (2008) fue traducido del idioma inglés al español siguiendo las recomendaciones propuestas por Geisinger (1994) sobre la traducción y adaptación de instrumentos de evaluación de diferentes culturas y lenguajes.

Antes de iniciar la recolección de datos, se contó con la autorización de la Dirección de Educación a Distancia de la Fundación. Los datos fueron recogidos por medio de la plataforma SurveyMonkey ${ }^{\mathrm{TM}}$, la cual permite administrar cuestionarios de encuestas a través de Internet. Dos semanas antes de finalizar el periodo de clases, los 125 estudiantes matriculados en el programa de teología recibieron una invitación, vía correo electrónico, para participar voluntariamente en el estudio. En el mismo correo se incluyó la dirección electrónica del cuestionario, el cual fue configurado para que sea respondido una sola vez por cada participante. Para el análisis de los datos se usó el programa estadístico SPSS $^{\mathrm{TM}}$ versión 20 para computadoras Apple ${ }^{\mathrm{TM}}$.

\section{Mediciones}

La medición en este estudio fue el nivel de satisfacción de los estudiantes de cursos virtuales. Para esto, se usó el cuestionario de factores críticos de Sun et al. (2008). Este cuestionario midió la relación de trece factores asociados a la experiencia de aprendizaje en cursos virtuales como variables independientes y la satisfacción estudiantil como variable dependiente.

Las variables independientes medidas son: actitud de los estudiantes hacia las computadoras, ansiedad de los estudiantes por el uso de computadoras, autoeficacia de los estudiantes en el uso de Internet, respuesta oportuna del docente, actitudes del docente hacia el e-learning, flexibilidad del curso, calidad del curso, calidad de la tecnología, calidad de Internet, percepción de utilidad del sistema virtual, percepción de facilidad de uso del sistema virtual, diversidad de la evaluación del aprendizaje y percepción de interacción con otros. A diferencia de la investigación original, en este estudio todas las escalas que componen el cuestionario fueron medidas con una escala tipo Likert de siete puntos, ordenadas desde 'totalmente en desacuerdo' (marcado con 1) hasta 'totalmente de acuerdo' (marcado con 7). 


\section{RESULTADOS}

\section{Análisis confiabilidad del instrumento}

El cálculo de la consistencia interna se hizo con el programa SPSS ${ }^{\mathrm{TM}}$. Se realizó el análisis de confiabilidad mediante el coeficiente Alfa de Cronbach para el instrumento completo y para las escalas que lo componen. En el cálculo de todo el instrumento se incluyeron los 73 reactivos y se obtuvo un Alfa de 0.92.

La tabla 2 presenta los cálculos del Alfa de las 11 escalas. Como se puede observar, la mayoría de las escalas alcanzaron el mínimo de confiabilidad aceptable de 0.7 (Kline, 1999): actitud del estudiante hacia las computadoras $=0.73$; la ansiedad del estudiante por el uso de computadoras $=0.84$; autoeficacia del estudiante en el uso de Internet $=0.93$; la flexibilidad del curso $=0.76$; calidad del curso $=0.85$; percepción de utilidad del sistema virtual $=0.86$, percepción de facilidad de uso del sistema virtual $=0.77$; percepción de la interacción con otros $=0.85$; percepción de satisfacción de los estudiantes de cursos virtuales $=0.81$. Las escalas que no alcanzaron el mínimo aceptable son las de calidad de Internet 0.59 y de calidad tecnológica 0.66 .

\section{Análisis de correlaciones y regresión}

Sellevó a cabo el análisis de correlación y luego de regresión por el método de pasos sucesivos entre la percepción de satisfacción de los estudiantes de cursos virtuales como variable dependiente y la actitud del estudiante hacia las computadoras, la ansiedad del estudiante por el uso de computadoras, la autoeficacia del estudiante en el uso de Internet, la respuesta oportuna del docente, la actitud del docente hacia el e-learning, la flexibilidad del curso, la calidad del curso, la calidad tecnológica, la calidad de Internet, la percepción de utilidad del sistema virtual, la percepción de facilidad de uso del sistema virtual, la diversidad en la evaluación y la percepción de la interacción con otros como variables independientes.

Para contrastar las hipótesis se calculó el coeficiente de correlación. Los resultados obtenidos se muestran en la tabla 2. Como se puede observar, se confirmaron 12 de las 13 hipótesis planteadas. Se encontró que la actitud de los estudiantes hacia las computadoras $(r=.26 ; p<0.01)$, la autoeficacia de los estudiantes por el uso de Internet $(r=.37 ; p<0.01)$, la respuesta oportuna del docente $(r=.26 ; p<0.01)$, las actitudes del docente hacia el e-learning $(r=.43 ; p<0.01)$, la flexibilidad del curso $(r=.57 ; p<0.01)$, la calidad del curso $(r=.52 ; p<0.01)$, la calidad de la tecnología $(r=.33 ; p<0.01)$, la calidad de Internet $(r=.35 ; p<0.01)$, la percepción de utilidad del sistema $(r=.26 ; p<0.01)$, la percepción de facilidad de uso del sistema $(r=.26 ; p<0.01)$, la diversidad de la evaluación del aprendizaje $(r=.41 ; p<$ o.01) y la percepción de interacción con otros $(r=.45 ; p<0.01)$ están positivamente 
correlacionadas con la percepción de satisfacción de los estudiantes de los cursos virtuales. Se encontró que la ansiedad del estudiante por el uso de computadoras está negativamente correlacionada con la percepción de satisfacción estudiantil ( $r=$ -.17), pero que no es significativa. La tabla 3 presenta un resumen de las pruebas que indica la aceptación/rechazo de las hipótesis.

Con estos resultados se procedió a probar el modelo mediante la regresión. Se realizaron los cálculos para asegurarse de no violar los supuestos de este método. Se solicitó un gráfico P-P normal de regresión de residuo tipificado y se observó que no hay desviaciones importantes. Además, esta normalidad se observó en el gráfico de dispersión, el cual no mostró patrones claros o sistemáticos de los residuos. Se usó el Índice de Condición para evaluar la multicolinealidad entre las variables independientes, resultando en 25.22 sin que alcance el valor moderado de 30 sugerido por Belsley, Kuh y Welsch (2005, p. 105). También se solicitó la prueba de Durbin-Watson para detectar correlaciones seriales entre errores. Esta prueba produjo el valor de 1.73 que según Field (2013, p. 311) por su cercanía al valor 2 indica que los residuos están incorrelacionados. 


\section{J. ZAMBRANO}

FACTORES PREDICTORES DE LA SATISFACCIÓN DE ESTUDIANTES DE CURSOS VIRTUALES

\begin{tabular}{|c|c|c|c|c|c|c|c|c|c|c|c|c|c|c|c|}
\hline$\stackrel{\Re}{\sim}$ & 1 & 1 & 1 & 1 & 1 & 1 & 1 & 1 & 1 & 1 & 1 & 1 & 1 & * & \\
\hline શ & 1 & 1 & 1 & 1 & 1 & 1 & 1 & 1 & 1 & 1 & 1 & 1 & $\stackrel{*}{*}$ & * & \\
\hline 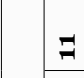 & 1 & 1 & 1 & 1 & 1 & 1 & 1 & 1 & 1 & 1 & 1 & $?$ & 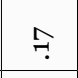 & * & \\
\hline$\stackrel{-}{\circ}$ & 1 & 1 & 1 & 1 & 1 & 1 & 1 & 1 & 1 & 1 & $\begin{array}{l}\text { * } \\
\text { ? } \\
\\
\end{array}$ & $\stackrel{10}{7}$ & 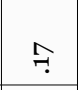 & *ै & \\
\hline$a$ & 1 & 1 & 1 & 1 & 1 & 1 & 1 & 1 & 1 & *ै & "ै? & $\stackrel{N}{7}$ & " సิ & $\begin{array}{l}\text { * } \\
\text { ? } \\
?\end{array}$ & \\
\hline$\infty$ & 1 & 1 & 1 & 1 & 1 & 1 & 1 & 1 & *ैं & * & ?ִ & 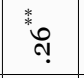 & 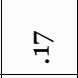 & *ึ & \\
\hline$\Lambda$ & 1 & 1 & 1 & 1 & 1 & 1 & 1 & "ิ & * ִָ & $\begin{array}{l}* \\
\text { * } \\
\text { ஸ̣ } \\
\end{array}$ & 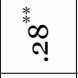 & $\stackrel{*}{*}$ & *ै & * * & \\
\hline 0 & 1 & 1 & 1 & 1 & 1 & 1 & $\stackrel{*}{*}$ & 类 & : & $\begin{array}{l}* \\
\text { * } \\
\text { ஸे } \\
\end{array}$ & 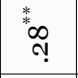 & "? & *ै & ? & 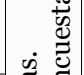 \\
\hline 10 & 1 & 1 & 1 & 1 & 1 & 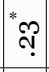 & *ैo & : & ๖̣ & $\overbrace{i}^{\infty}$ & ô & * & *ैo & *ै? & $\begin{array}{ll} & 0 \\
0 & 0 \\
0 & 0\end{array}$ \\
\hline$\tau$ & 1 & 1 & 1 & 1 & "ै? & * & * & O̦ & 0 & है & ơ & ". & *ै & "พ & $\begin{array}{l}0 \\
0.0 \\
0 \\
0\end{array}$ \\
\hline 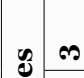 & 1 & 1 & 1 & $\begin{array}{l}\infty \\
0 \\
i\end{array}$ & $\begin{array}{l}0 \\
\text { ô. } \\
\text { i }\end{array}$ & \% & * & * & * & * $\stackrel{\text { * }}{+}$ & "* & " & $\stackrel{+}{0}$ & "ै & \begin{tabular}{ll}
$v$ & \multicolumn{2}{c}{} \\
2 & 0 \\
$*$ & 0
\end{tabular} \\
\hline N| & 1 & 1 & *ڤ్ & $\stackrel{?}{?}$ & $\exists$ & $\widehat{\imath}$ & $\begin{array}{l}7 \\
i \\
i\end{array}$ & \begin{tabular}{c}
$*$ \\
\multirow{2}{*}{} \\
$\stackrel{1}{1}$ \\
1
\end{tabular} & $\begin{array}{l}0 \\
7 \\
1\end{array}$ & \% & * & $\stackrel{8}{0}$ & ô. & $\hat{\imath}$ & 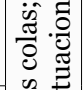 \\
\hline \begin{tabular}{|l|l} 
& \\
& 0 \\
0
\end{tabular} & 1 & * ִָ & ? & $\hat{\hat{o}}$ & o & ฟิ & $\stackrel{\oplus}{?}$ & * & *ै & ". & ". & $\stackrel{10}{\nearrow}$ & $\stackrel{0}{0}$ & "ִ̣ & 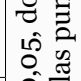 \\
\hline 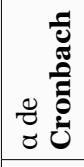 & 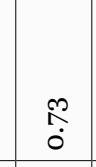 & $\begin{array}{l}\infty \\
0 \\
0\end{array}$ & 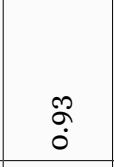 & 1 & 1 & $\begin{array}{l}2 \\
0 \\
0\end{array}$ & $\begin{array}{c}10 \\
0 \\
0 \\
\end{array}$ & $\begin{array}{l}0 \\
0 \\
0 \\
0\end{array}$ & \begin{tabular}{l}
0 \\
\multirow{2}{0}{} \\
0
\end{tabular} & $\begin{array}{l}\infty \\
\infty \\
0 \\
0\end{array}$ & $\hat{0}$ & 1 & $\begin{array}{l}10 \\
0 \\
0\end{array}$ & $\begin{array}{l}-1 \\
0 \\
0\end{array}$ & 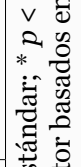 \\
\hline 당 & $\stackrel{v}{0}$ & مِ & $\begin{array}{c}\infty \\
\infty \\
0 \\
0\end{array}$ & $\begin{array}{l}\dot{I} \\
\text { ì }\end{array}$ & 족 & \begin{tabular}{|c|}
12 \\
$\hat{0}$ \\
0
\end{tabular} & $\stackrel{10}{\leftrightarrows}$ & $\begin{array}{l}\hat{\sigma} \\
\dot{0} \\
\end{array}$ & $\underset{+}{\stackrel{T}{ }}$ & $\begin{array}{l}\infty \\
0 \\
0\end{array}$ & $\begin{array}{l}\infty \\
0 \\
0\end{array}$ & 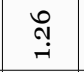 & 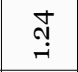 & 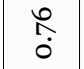 & : \\
\hline$\Sigma$ & $\begin{array}{l}\text { L̊ } \\
\text { if }\end{array}$ & 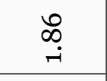 & $\begin{array}{l}\dot{T} \\
\dot{1} \\
\end{array}$ & $\stackrel{\mathscr{7}}{\dot{\gamma}}$ & $\underset{⿱ ㇒}{+}$ & $\begin{array}{l}\overrightarrow{0} \\
\dot{\delta} \\
\dot{\omega}\end{array}$ & \begin{tabular}{l}
$\infty$ \\
\multirow{1}{0}{} \\
$\dot{1}$ \\
\end{tabular} & $\begin{array}{l}\hat{\sigma} \\
\hat{\sigma} \\
\end{array}$ & $\begin{array}{l}\stackrel{0}{\circ} \\
\dot{1}\end{array}$ & 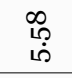 & $\begin{array}{l}\infty \\
10 \\
10 \\
10\end{array}$ & $\begin{array}{l}\hat{1} \\
10 \\
10\end{array}$ & $\begin{array}{l}\vec{\sigma} \\
\dot{m}\end{array}$ & $\begin{array}{l}\mathscr{2} \\
\dot{1}\end{array}$ & $\begin{array}{cc}5 & 2 \\
0 & 0 \\
0 & 0 \\
0 & 0 \\
0\end{array}$ \\
\hline 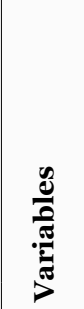 & 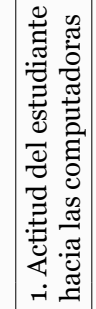 & 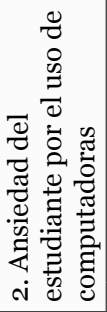 & 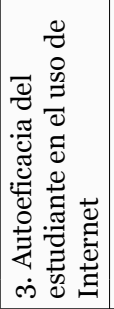 & 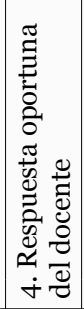 & 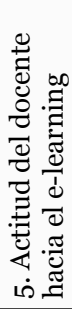 & 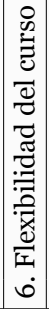 & 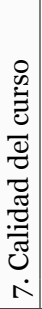 & 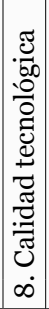 & 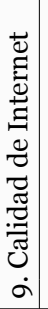 & 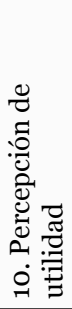 & 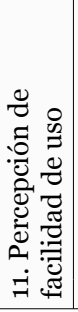 & 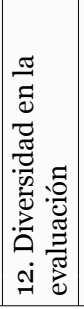 & 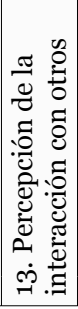 & 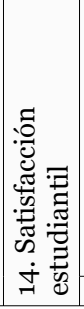 & 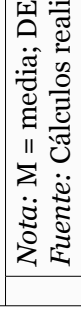 \\
\hline
\end{tabular}


Tabla 3. Pruebas de las hipótesis planteadas

\begin{tabular}{|l|l|}
\hline \multicolumn{1}{|c|}{ Hipótesis planteadas } & Aceptación \\
\hline $\begin{array}{l}\text { H1. La actitud de los estudiantes hacia las computadoras está positivamente } \\
\text { relacionada con su percepción de satisfacción. }\end{array}$ & Sí \\
\hline $\begin{array}{l}\text { H2. La ansiedad de los estudiantes por el uso de computadoras está } \\
\text { negativamente relacionada con su percepción de satisfacción. }\end{array}$ & No \\
\hline $\begin{array}{l}\text { H3. La autoeficacia de los estudiantes por el uso de Internet está } \\
\text { positivamente relacionada con su percepción de satisfacción. }\end{array}$ & Sí \\
\hline $\begin{array}{l}\text { H4. La respuesta oportuna del docente está positivamente relacionada con } \\
\text { la percepción de satisfacción estudiantil. }\end{array}$ & Sí \\
\hline $\begin{array}{l}\text { H5. Las actitudes del docente hacia el e-learning están positivamente } \\
\text { relacionadas con la percepción de satisfacción estudiantil. }\end{array}$ & Sí \\
\hline $\begin{array}{l}\text { H6. La flexibilidad del curso está positivamente relacionada con la } \\
\text { percepción de satisfacción estudiantil. }\end{array}$ & Sí \\
\hline $\begin{array}{l}\text { H7. La calidad del curso está positivamente relacionada con la percepción } \\
\text { de satisfacción estudiantil. }\end{array}$ & Sí \\
\hline $\begin{array}{l}\text { H8. La calidad de la tecnología está positivamente relacionada con la } \\
\text { percepción de satisfacción estudiantil. }\end{array}$ & Sí \\
\hline $\begin{array}{l}\text { H9. La calidad de la Internet está positivamente relacionada con la } \\
\text { percepción de satisfacción estudiantil. }\end{array}$ & Sí \\
\hline $\begin{array}{l}\text { H1o. La percepción de utilidad del sistema está positivamente relacionada } \\
\text { con la percepción de satisfacción estudiantil. }\end{array}$ & Sí \\
\hline $\begin{array}{l}\text { H11. La percepción de facilidad de uso del sistema está positivamente } \\
\text { relacionada con la percepción de satisfacción estudiantil. }\end{array}$ & Sí \\
\hline $\begin{array}{l}\text { H12. La diversidad de la evaluación del aprendizaje está positivamente } \\
\text { relacionada con la percepción de satisfacción estudiantil. }\end{array}$ \\
\hline $\begin{array}{l}\text { H13. La percepción de la interacción con otros está positivamente } \\
\text { relacionada con su percepción de satisfacción. }\end{array}$ & Sí \\
\hline fína
\end{tabular}

La tabla 4 muestra los resultados de la regresión con pasos sucesivos. La regresión $R$ fue significativamente diferente de cero, $\mathrm{F}(4,97)=23.59, \mathrm{p}<.001$. De las trece variables quedaron cuatro en el siguiente orden: flexibilidad del curso, actitud docente hacia el e-learning, autoeficacia del estudiante en el uso de Internet y percepción de la interacción con otros. El $R^{2}$ del modelo compuesto por la flexibilidad del curso es .326 y el $R^{2}$ corregida .320; la variable flexibilidad es el factor predictor más fuerte porque produce un tercio de la variabilidad de la satisfacción percibida por los estudiantes de los cursos virtuales. El modelo compuesto por la flexibilidad del curso 
y la actitud del docente hacia el e-learning obtuvo un $R^{2}$ de .419 y un $R^{2}$ ajustada de .407. La flexibilidad, la actitud del docente y la autoeficacia del estudiante en el uso de Internet tuvieron un $R^{2}$ de .469 y un $R^{2}$ ajustada .453. Y el modelo que combina las variables flexibilidad del curso, actitud docente hacia el e-learning, autoeficacia del estudiante en el uso de Internet y la percepción de la interacción con otros dio como resultado un $R^{2}$ de .493 y el valor de $R^{2}$ ajustada de .472 . Este valor indica que casi la mitad de la variabilidad de la satisfacción percibida por los estudiantes de cursos virtuales se puede predecir por la combinación de estas últimas cuatro variables.

Tabla 4. Resultados de la regresión entre las variables independientes y la satisfacción estudiantil

\begin{tabular}{|c|c|c|}
\hline \multirow[t]{2}{*}{ Variables independientes } & \multicolumn{2}{|c|}{$\begin{array}{c}\text { Variable dependiente: satisfacción } \\
\text { percibida por el estudiante de cursos } \\
\text { virtuales }\end{array}$} \\
\hline & Valor $\beta$ & Valor t \\
\hline Actitud del estudiante hacia las computadoras & 0.09 & 1.04 \\
\hline Ansiedad del estudiante por el uso de computadoras & -0.04 & -0.56 \\
\hline Autoeficacia del estudiante en el uso de Internet & 0.25 & $3.20^{* *}$ \\
\hline Respuesta oportuna del docente & -0.08 & -0.88 \\
\hline Actitud del docente hacia el e-learning & 0.27 & $3.24^{* *}$ \\
\hline Flexibilidad del curso & 0.36 & $4 \cdot 32^{* * *}$ \\
\hline Calidad del curso & 0.17 & 1.92 \\
\hline Calidad tecnológica & 0.05 & 0.51 \\
\hline Calidad de Internet & 0.07 & 0.78 \\
\hline Percepción de utilidad & 0.02 & 0.19 \\
\hline Percepción de facilidad de uso & 0.02 & 0.19 \\
\hline Diversidad en la evaluación & 0.04 & 0.41 \\
\hline Percepción de la interacción con otros & 0.19 & $2.16^{*}$ \\
\hline$F(4,97)$ & $23.59^{* * *}$ & \\
\hline$R$ & $0.702^{*}$ & \\
\hline$R^{2}$ & 0.493 & \\
\hline$R^{2}$ corregida & 0.472 & \\
\hline Índice de Condición & 25.22 & \\
\hline Durbin-Watson & 1.73 & \\
\hline $\begin{array}{l}\text { Nota: }{ }^{*} \mathrm{p}<.05 ; * * \mathrm{p}<.01 ;{ }^{* * * *} \mathrm{p}<.001 . \\
\text { Fuente: Cálculos realizados por el autor }\end{array}$ & & \\
\hline
\end{tabular}




\section{DISCUSIÓN}

La educación virtual tiene muchas ventajas en la medida en que el diseño instruccional y las tecnologías tengan en cuenta los factores que afectan el rendimiento y la satisfacción estudiantil. Este estudio ofrece un marco de análisis y un instrumento que ayuda a comprender qué factores afectan positiva y negativamente a la satisfacción estudiantil, con el propósito de emprender mejoras en el actual desarrollo del curso virtual y en futuros diseños. Aunque la percepción de satisfacción con el curso virtual no está directamente relacionada con el desempeño académico del estudiante, sí está relacionada con dimensiones afectivas que al parecer influyen en su retención en el curso virtual y su expectativa general sobre la modalidad de estudios (García Aretio, Ruíz y Domínguez, 2007). Por esta razón, se debe tener en cuenta no solamente el rendimiento cognitivo del estudiante, sino también su satisfacción con toda la experiencia de aprender virtualmente. El modelo y el cuestionario usado en este estudio ofrecen una visión multifactorial que contribuye tanto a administradores como a profesores a tomar decisiones oportunas y acciones efectivas para mejorar la experiencia de los estudiantes en los cursos virtuales.

Como se pudo ver en el apartado de los resultados, el cuestionario demostró ser altamente confiable. Aunque con un valor ligeramente inferior en las escalas sobre la calidad de Internet y sobre la calidad tecnológica. El cuestionario en su totalidad, así como las otras nueve escalas que lo conforman, resultaron tener alta consistencia interna. La confiabilidad en este estudio es similar a la obtenida por Sun et al. (2008). En la investigación de Sun, la escala con mayor confiabilidad fue la satisfacción percibida por los estudiantes $=0.93$, y la de menor confiabilidad y la única por debajo del mínimo aceptable fue la calidad de Internet $=0.50$. Estos resultados ofrecen más evidencia para que los administradores de programas virtuales universitarios usen el modelo y el cuestionario con sus propios estudiantes.

Algunos de los factores significativos identificados por esta investigación coinciden con los encontrados en el estudio original. Sun y sus colaboradores evidenciaron que siete de las trece hipótesis tienen correlación significativa. Las variables correlacionadas fueron: la ansiedad de los estudiantes por el uso de computadoras, las actitudes del docente hacia el e-learning, la flexibilidad del curso, calidad del curso, la percepción de utilidad del sistema virtual, la percepción de facilidad de uso del sistema virtual y la diversidad de la evaluación del aprendizaje. En la presente investigación, en cambio, solamente la ansiedad de los estudiantes por el uso de computadoras no fue significativa.

$\mathrm{El}$ análisis correlacional demuestra que los factores tomados de forma individual predicen la dirección de la satisfacción estudiantil. Considerando la prescripción de Cohen (1988), los factores con mayor fuerza relacionados a la satisfacción estudiantil son la flexibilidad del curso y la calidad del curso. Sun y sus colaboradores también encontraron fuerte correlación en el factor de la calidad del curso y la flexibilidad alcanzó mediana fuerza predictiva. En ambos estudios se puede observar que la 
calidad del curso es un aspecto crítico para los estudiantes. La percepción de este aspecto depende de la experiencia previa que ellos tienen con otros cursos virtuales y presenciales (Song, Singleton, Hill y Koh, 2004). Por esta razón urge explorar e identificar los factores particulares que conforman estas expectativas según la percepción estudiantil (véase el trabajo de Jung, 2011 por ejemplo), a fin de encontrar las visiones conflictivas entre los diseñadores, profesores y estudiantes para realizar mejoras más informadas.

La alta correlación de la flexibilidad del curso es un indicador importante de una de las ventajas de aprender a través de cursos virtuales. García Aretio (2001) ya ha anotado las diferentes barreras para el aprendizaje, tales como las espaciotemporales y cómo la flexibilidad de los cursos virtuales contribuye a superarlas. Los estudiantes relacionan la flexibilidad del curso con su satisfacción debido a que aprender mediante cursos virtuales les permite organizar efectivamente las diferentes actividades propias de la vida adulta, tales como el trabajo, la familia u otros estudios. Ante las presiones de la vida adulta, la flexibilidad permite a los estudiantes la autonomía necesaria para programar adecuadamente los ritmos, momentos y formas de aprender. Por estas razones, es imperativo que los administradores tengan presente el grado de flexibilidad que ofrece el curso, así como las actividades de aprendizaje. Al aplicar el instrumento de este estudio, de manera sistemática, los administradores podrán conocer el efecto de los cambios en la flexibilidad del curso sobre la percepción estudiantil.

El análisis de regresión de Sun y sus colaboradores encontró siete factores predictores, dos de los cuales concuerdan con este estudio: la actitud del instructor hacia el e-learning y la flexibilidad del curso. La significatividad de estos dos factores pone de manifiesto la relevancia del trabajo de los administradores y diseñadores instruccionales de orientar a los docentes sobre el uso adecuado de las herramientas del sistema y sobre la conducción del curso virtual. Los instructores cumplen un importante rol en la satisfacción estudiantil, por lo que los administradores deben examinar con mucho más detalle de qué manera los diálogos entre el instructor y los estudiantes están afectando las actitudes de los estudiantes.

La importancia del trabajo del instructor y su relación con la satisfacción estudiantil ha sido observada en varios estudios y desde diversas perspectivas. Allen et al. (2013), por ejemplo, encontró que la percepción de la interacción predice la satisfacción estudiantil. Otros como Shin (2003) desde la perspectiva transaccional, Akyol y Garrison (2010) desde el modelo de la comunidad de indagación, así como Kirschner, Kreijns, Phielix y Fransen (2014) desde el modelo de conciencia del comportamiento social han mostrado empíricamente cómo influye el trabajo docente en el aprendizaje y satisfacción de los estudiantes. Kirschner, Kreijns, Phielix y Fransen (2014) encontraron que la percepción del trabajo docente no solamente afecta al rendimiento cognitivo estudiantil, sino también a otros factores sociales y emocionales relacionados con la experiencia de aprender vía Internet. Por esta razón, los administradores y diseñadores deben poner mucha atención a la 
gestión didáctica de los cursos virtuales, monitorear el desempeño y la satisfacción estudiantil durante el proceso de aprendizaje y hacer cambios oportunos tanto en el diseño instruccional como en la actuación docente.

Para la mejora continua del modelo predictivo y del instrumento, se recomienda realizar otros estudios que repliquen el procedimiento con otras poblaciones de estudiantes, diferenciando el tipo de carrera universitaria, nivel de conocimiento (e.g. novatos versus avanzados) y curricular (e.g. grado o posgrado), grupos culturales y tipos de material instruccional (e.g. curso mayormente basado en texto, o en vídeo). También se sugiere introducir otras variables predictoras tales como las demandas cognitivas de las tareas de aprendizaje o el tipo de diseño instruccional utilizado en el curso (van Mierlo, Jarodzka, Kirschner y Kirschner, 2012).

Una limitación de esta investigación y también de Sun y sus colaboradores, es el tamaño de la muestra. El método de regresión por pasos sucesivos exige que las Jhipótesis sean contrastadas con muchos más casos. Tabachnick y Fidell (2013, p. 124) recomiendan una ratio de 40 casos como mínimo por cada variable independiente para que los resultados no den lugar a generalizaciones más allá de la muestra. Por esto se advierte que los futuros estudios de réplica amplíen la muestra con al menos 520 casos.

\section{REFERENCIAS BIBLIOGRÁFICAS}

Akyol, Z., y Garrison, D. R. (2010). Community of inquiry in adult online learning: Collaborative-constructivist approaches. En T. T. Kidd y J. Keengwe (Ed.), Adult learning in the digital age: Perspectives on online technologies and outcomes (52-66). Hershey, PA: Information Science Reference.

Allen, M., Bourhis, J., Burrell, N., y Mabry, E. (2002). Comparing student satisfaction with distance education to traditional classrooms in higher education: A metaanalysis. American Journal of Distance Education, 16(2), 83-97. doi: 10.1207/ S15389286AJDE1602_3

Allen, M., Omori, K., Burrell, N., Mabry, E., y Timmerman, E. (2013). Satisfaction with distance education. In M. G. Moore (Ed.), Handbook of distance education. 3ra. ed. (143-154). Nueva York: Routledge.

Belsley, D. A., Kuh, E., y Welsch, R. E. (2005). Regression diagnostics:
Identifying influential data and sources of collinearity, 571, John Wiley \& Sons.

Bolliger, D. U., y Martindale, T. (2004). Key factors for determining student satisfaction in online courses. International Journal on E-Learning, 3(1), 61-67.

Bray, E., Aoki, K., y Dlugosh, L. (2008). Predictors of learning satisfaction in Japanese online distance learners. The International Review of Research in Open and Distributed Learning, 9(3).

Cohen, J. (1988). Statistical power analysis for the behavioral sciences. 2nd ed. Hillsdale, N.J.: L. Erlbaum Associates.

Drouin, M. A. (2008). The relationship between students' perceived sense of community and satisfaction, achievement, and retention in an online course. Quarterly Review of Distance Education, 9(3), 267-284.

Field, A. (2013). Discovering statistics using IBM SPSS statistics. 4ta. ed. Los Angeles: Sage. 
García Aretio, L. (2001). La educación a distancia: De la teoría a la práctica. Barcelona: Editorial Ariel.

García Aretio, L., Ruíz, M., y Domínguez, D. (2007). De la educación a distancia a la educación virtual. Barcelona: Editorial Ariel.

Geisinger, K. F. (1994). Cross-cultural normative assessment: Translation and adaptation issues influencing the normative interpretation of assessment instruments. Psychological assessment, 6(4), 304. doi: 10.1037/10403590.6.4.304.

Jung, I. (2011). The dimensions of e-learning quality: From the learner's perspective. Educational Technology Research and Development, 59(4), 445-464. doi: 10.1007/s11423-010-9171-4.

Kirschner, P. A., Kreijns, K., Phielix, C., $\mathrm{y}$ Fransen, J. (2014). Awareness of cognitive and social behaviour in a CSCL environment. Journal of Computer Assisted Learning, 59-77. doi: 10.1111/ jcal.12084.

Kline, P. (1999). The handbook of psychological testing. 2da. ed. Londres: Routledge.

Moore, J. C., y Shelton, K. (2014). The Sloan Consortium pillars and quality scorecard. In K. Shattuck (Ed.), Assuring quality in online education: Practices and processes at the teaching, resource, and program levels (pp. 40-49). Sterling, Virginia: Stylus Publishing, LLC.

Moore, M. G. (Ed.). (2013). Handbook of distance education. 3ra. ed. Nueva York: Routledge.

Peralta Castro, R., Escobar Jurado, S. I., Mora Rodríguez, J. R., Martínez González, C., y Rocío Velandia, L. S. (2014). Caracterización de los factores de la deserción en la UNAD. Informe final de investigación. Universidad Nacional Abierta y a Distancia, Asociación Panamericana de Instituciones de Crédito
Educativo. Bogotá. Recuperado de http:/ goo.gl/lyVxVu

Rubio Gómez, M. J. (2003). Memoria. Proyecto: Centro Virtual para el Desarrollo de Estándares de Calidad para la Educación Superior a Distancia en América Latina y el Caribe. Instituto Latinoamericano y del Caribe de Calidad en Educación Superior a Distancia. Loja, Ecuador. Recuperado de http://gdr1. utpl.edu.ec/centrovirtual/documentos/ memorias.pdf

Rudestam, K. E., y Schoenholtz-Read, J. (2010). Handbook of online learning. 2da. ed. Thousand Oaks, Calif.: SAGE Publications.

Sanjuán Gómez, G., Gómez Martínez, M., Rabell Piera, O., Arcia Arcia, L., y Morales Velázquez, I. C. (2011). Resultados preliminares del grado de satisfacción con el empleo del aula virtual de la Facultad de Ciencias Médicas General Calixto García. Revista Habanera de Ciencias Médicas, 1O(1), 114-125.

Shin, N. (2003). Transactional presence as a critical predictor of success in distance learning. Distance Education, 24(1), 6986. doi: 10.1080/01587910303048.

Simpson, O. (2003). Student retention in online, open, and distance learning. Sterling VA: Kogan Page.

Song, L., Singleton, E. S., Hill, J. R., y Koh, M. H. (2004). Improving online learning: Student perceptions of useful and challenging characteristics. The Internet and Higher Education, 7(1), 59-70. doi: 10.1016/j.iheduc.2003.11.003.

Sun, P.-C., Tsai, R. J., Finger, G., Chen, Y.-Y., y Yeh, D. (2008). What drives a successful e-learning? An empirical investigation of the critical factors influencing learner satisfaction. Computers \& Education, 5O(4), 1183-1202. doi: 10.1016/j. compedu.2006.11.007.

Swan, K. (2001). Virtual interaction: Design factors affecting student satisfaction and perceived learning in asynchronous online 
courses. Distance Education, 22(2), 306331. doi: 10.1080/0158791010220208

Tabachnick, B. G., y Fidell, L. S. (2013). Using multivariate statistics. 6ta. ed. Boston: Pearson Education.

Van Mierlo, C. M., Jarodzka, H., Kirschner, F., y Kirschner, P. A. (2012). Cognitive load theory in e-learning. In Z. Yan (Ed.), Encyclopedia of Cyber Behavior, 3, (11781211). IGI Global.
Williams, S. L. (2006). The effectiveness of distance education in allied health science programs: A meta-analysis of outcomes. American Journal of Distance Education, 2O(3), 127-141. doi: 10.1207/ s15389286ajde2003_2.

Zambrano R., J. (2012). La docencia en la sociedad red: Apuntes para la formación de docencia virtual, 1, Quito: Corporación para el Desarrollo de la Educación Universitaria.

\section{PERFIL ACADÉMICO Y PROFESIONAL DEL AUTOR}

Jimmy Zambrano Ramírez. Docente e investigador del Instituto Superior Tecnológico Rumiñahui (Ecuador). Candidato al $\mathrm{PhD}$ en la Open University of the Netherlands y estudiante del Doctorado en Educación en la Universidad Católica Andrés Bello de Venezuela, se encuentra en proceso de investigación de la efectividad del aprendizaje colaborativo desde la perspectiva de la teoría de la carga cognitiva.

E-mail: info@jimmyzambrano.com

\section{DIRECCIÓN DEL AUTOR}

Instituto Superior Tecnológico Rumiñahui Avda. Atahualpa 1701 y Calle 8 de Febrero Sangolquí (Ecuador)

Fecha de recepción del artículo: 10/08/2015

Fecha de aceptación del artículo: 22/10/2015

\section{Como citar este artículo:}

Zambrano Ramírez, J. (2016). Factores predictores de la satisfacción de estudiantes de cursos virtuales RIED. Revista Iberoamericana de Educación a Distancia, 19(2), pp. 217-235. doi: http://dx.doi.org/10.5944/ried.19.2.15112 